\title{
Some Problems of Elementary Particles Physics and Gilbert's Postulate
}

\author{
Boris V. Vasiliev \\ Independent Researcher, Russia \\ Email: bv.vasiliev@yandex.com
}

How to cite this paper: Vasiliev, B.V. (2016) Some Problems of Elementary Particles Physics and Gilbert's Postulate. Journal of Modern Physics, 7, 1874-1888. http://dx.doi.org/10.4236/jmp.2016.714166

Received: August 11, 2016

Accepted: October 15, 2016

Published: October 19, 2016

Copyright () 2016 by author and Scientific Research Publishing Inc. This work is licensed under the Creative Commons Attribution International License (CC BY 4.0).

http://creativecommons.org/licenses/by/4.0/

\begin{abstract}
William Gilbert formulated over 400 years ago a postulate that can be considered as the main principle of modern natural sciences [1]: All theoretical constructs that claim to be scientific must be verified and confirmed experimentally. Despite of past centuries, this principle has not lost its relevance today. In the modern physics there are some conventional theories, which do not satisfy to Gilbert's postulate [2]. In physics of microcosm there are models which cannot be compared with the measurement data as they do not allow to calculate the basic characteristic parameters (such as masses or magnetic moments) of elementary particles. In this article an alternative approach to these problems is considered. It is shown that an attraction in the proton-neutron pair can occur due to the exchange of relativistic electron. The estimation of this exchange energy is in agreement with the experimental values of the binding energy of some light nuclei. At that neutron is regarded as a composite corpuscule consisting of proton and relativistic electron that allows predicting the neutron magnetic moment, its mass and energy of its decay. It is shown that the standard Maxwell's theory of electromagnetic field describes a possibility to initiate in free space (in empty ether) a magnetic $\gamma$-quantum (a splash of magnetic field), devoid of the electric component and having spin $\hbar / 2$. Since magnetic monopoles do not exist, a characteristic feature of the magnetic $\gamma$-quantum is the weakness of its interaction with matter, which is many orders of magnitude smaller than that of the electromagnetic wave. These properties suggest that the magnetic $\gamma$-quantum can be identified with neutrinos. On this basis, we get a fresh look on the nature of $\pi$-mesons and $\mu$-mesons and calculate their masses.
\end{abstract}

\section{Keywords}

Proton, Neutron, Neutrino, Pion, Muon, Magnetic Moment, Mass 


\section{The Main Principle of Natural Sciences}

It may seem to our contemporaries, level of education of which corresponds to the development of science in the XXI century, that medieval science was generally concentrated in theology, astrology and alchemy. But this is not the case. The Middle Ages was a time of the development of the foundations of modern science.

Outstanding medieval scientist William Gilbert (1544-1603) introduced into scientific use the notion of electric and magnetic fields, taking the first step to understanding the nature of electromagnetism. He was first who tried to explain the nature of Earth's magnetic field.

But it seems that the most important of his contribution to science is the creation of postulate, which became the main principle of modern natural science studies $[1]^{1}$.

Gilbert principle is stated simply: All theoretical constructs that claim to be science must be verified and confirmed experimentally.

It seems that among our contemporary scholars there is no one who would have objected to it.

However, in the twentieth century a number of scientific constructs was created that have been accepted by the scientific community and still are dominant in their fields of knowledge, but at that they do not satisfy the principle of Gilbert.

\section{The Gilbert's Postulate and Modern Physics}

It should be emphasized that the vast majority of modern theoretical models adequately and accurately reflect the properties of matter and the laws of nature, their creation are conducted in full compliance with the Gilbert's postulate.

But in some cases, models developed by theorists at XX century were wrong [2].

Let us consider some problems of physics of elementary particles and their compliance with the Gilbert's postulate.

\section{Proton and Neutron}

\subsection{Proton and Neutron in Gell-Mann's Quark Model}

It seems that experts in particle physics at first proceeded from the assumption that our Maker at the creation of the World selected individually appropriate parameters for each elementary particle (charge, spin, mass, moment). Gell-Mann simplified this work. He developed a rule that a set of quarks defines total charge and spin of an formed elementary particle. But this rule do not spread on masses and magnetic moments of these particles (Figure 1).

The quark model is considered as the basis of modern elementary particle physics. The formation of this model in the chain of the structure of matter science seems quite consistent: All substances consist of atoms and molecules. Nuclei are central elements of atoms. They are composed of protons and neutrons, which in turn are composed of quarks.

${ }^{1}$ It is possible to assume that the idea of this principle, as they say, was in the air among the educated people of that time. But this principle was worded and came to us thanks to W. Gilbert. 


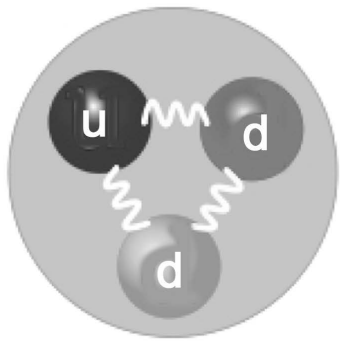

NEUTRON

Quark structure

charge $=0$

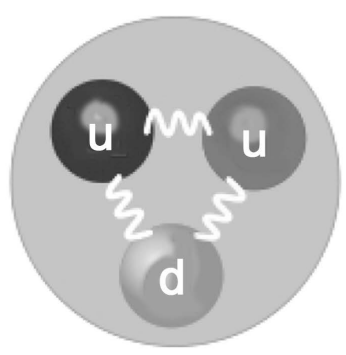

PROTON

Quark structure

charge $=+e$

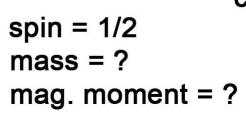

Figure 1. Protons and neutrons in Gell-Mann's quark model. The charges of quarks are chosen so that the transformation of neutron into proton carried by replacing one d-quark on u-quark. The Gell-Mann's model does not give predictions of masses and magnetic moments for proton and neutron.

The quark model implies that all elementary particles consist of quarks (except lightest particles). Accordingly to the Gell-Mann's quark model, to describe all of particle diversity, the quarks must have fractional electric charge $(1 / 3$ or $2 / 3 \mathrm{e})$ and other discrete properties, referred to as flavor, color, and others.

In the 60 years after the formulation of foundations of the quark model, many experimenters have tried to find particles with fractional charge. But unsuccessfully.

An existence of fractional charges in a free state did not found of the experimental verification. To explain this, it was suggested that a confinement is characteristic for quarks, ie, property, prohibiting them in any way to express themselves in a free state. The confinement was introduced to reconcile the model with the observed data (or rather with data of unobservation), but at the same time it withdraws quarks from subordination of Gilbert's postulate.

As such, the quark model with fractional charges claim on scientific validity without the confirmation of the measurement data. It should be noted that the quark model successfully describes some experiments on the scattering of particles at high energies, for example, the formation of jets or feature of the scattering of high-energy particles without destroying. However, this seems to be little in order to acknowledge the existence of quarks with fractional charge.

\subsection{Proton Consisting of Quarks with Integer Charge}

Let our goal to construct a model of proton from quarks with integer charges so that to predict its mass and magnetic moment. We assume that, as in Gell-Mann's model, proton consists of three quarks. But in our case two of them have charge $+\mathrm{e}$ and one $-\mathrm{e}$. Let these quarks do not possess their own spin, and their quantum motion expressed in their rotation around a common center on the circle of radius $\mathrm{R}$ (Figure 2). 


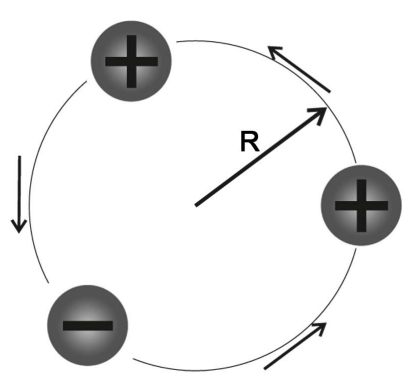

Figure 2. Proton consisting of two positively charged quarks and negatively charged one.

Let this movement of quarks is also carried out, as the motion of electron in the Bohr atom, i.e. the value of the radius $\mathrm{R}$ is determined by the fact that the length of the circumference $2 \pi R$ is equal to length of de Broglie waves of quark $\lambda_{D}$ :

$$
2 \pi R=\lambda_{D}=\frac{2 \pi \hbar}{p_{q}},
$$

where $p_{q}$ is quark momentum.

We assume that quarks have the same impulses $p_{q}$ and rotate in a single circle, so that equality (1) reduces to equation

$$
p_{q} R=\hbar
$$

Generalized moment of rotation (spin) of the system is made up of two components: mechanical torque is created by all three quarks $3 \boldsymbol{p}_{q} \times \boldsymbol{R}$, but magnetic field pulse is generated by charged by quark with non-compensated charge only $-\frac{e}{c} \boldsymbol{A}$ :

$$
\boldsymbol{s}=\boldsymbol{R}\left[3 \boldsymbol{p}_{q}-\frac{e}{c} \boldsymbol{A}\right] .
$$

Given that the magnetic vector potential is generated by the rotating charge

$$
\boldsymbol{A}=\frac{[\boldsymbol{\mu} \times \boldsymbol{R}]}{R^{3}}
$$

and the magnetic moment of a circular current

$$
\boldsymbol{\mu}=\frac{e}{2 c}[\boldsymbol{R} \times \boldsymbol{v}]
$$

we obtain invariant angular momentum (spin)

$$
s=\frac{\hbar}{2}\left(6-\frac{e^{2}}{\hbar c} \frac{1}{\sqrt{1-\beta^{2}}}\right)
$$

where $\beta=\frac{v}{c}$.

Based on the fact that the value of the proton spin is equal to $\hbar / 2$, we obtain

$$
\frac{\hbar}{2}=\frac{\hbar}{2}\left(6-\frac{\alpha}{\sqrt{1-\beta^{2}}}\right)
$$

where $\alpha=\frac{e^{2}}{\hbar c}$ is the fine structure constant. 
This equation leads us to the conclusion that quarks in a free state are positrons and electrons have mass $m_{e}$. While the mass of this quark-positron in the bounded state

$$
m_{q}=\frac{m_{e}}{\sqrt{1-\beta^{2}}}=\frac{5}{\alpha} m_{e} \simeq 685.2 m_{e} .
$$

Summary mass of all quarks

$$
3 m_{q} \simeq 2055 m_{e}
$$

that in satisfactory agreement with the measured value of proton mass:

$$
\frac{3 m_{q}}{M_{p}} \simeq 1.12
$$

Given the value of mass of charged quark (Equation (8)), the magnetic moment produced by this quark is found to be

$$
\mu_{q}=\frac{e \hbar}{2 m_{q} c}=\frac{M_{p}}{m_{q}} \frac{e \hbar}{2 M_{p} c} \approx 2.68 \mu_{N}
$$

(where $\mu_{N}=\frac{e \hbar}{2 M_{p} c}$ is the Borh's nuclear magnetic moment), which is close to the experimentally measured value of the magnetic moment of proton

$$
\mu_{p}=2.79 \mu_{N}
$$

Thus, the use of three quarks with integral charges makes it possible to build the model of proton, all parameters of which are close to observed data and there is no problem with the observability of these quarks in free state.

\subsection{Is Neutron an Elementary Particle?}

The Gell-Mann's quark model suggests that the neutron is an elementary particle in the sense that it consists of a different set of quarks than proton.

In the 30 s of the last century theoretical physicists concluded about elementary neutron without relying on measurement data, which were not at the time.

In order to explain results of measurements of neutron parameters-magnetic moment of neutron, its mass and energy of its decay-let's consider the electromagnetic model of the neutron, in which it is not an elementary particle ([3] [4]).

Let suppose that the neutron as well as Bohr's hydrogen atom consists of proton and electron which rotates round proton on a very small distance. Near proton the electron motion must be relativistic.

Consider the electromagnetic model of the neutron ([3] [4]) in more detail.

\subsection{The Electromagnetic Model of Neutron}

Let's consider the composite corpuscle, in which relativistic electron with mass $\gamma m_{e}$ and charge $-e$ spins around proton (where $\gamma=\frac{1}{\sqrt{1-\beta^{2}}}$ is relativistic factor, 
$\left.\beta=\frac{v}{C}\right)$.

Let there is a cylindrical coordinate system in which $\mathrm{z}$-axis coincides with the direction of the proton magnetic moment:

$$
\mathbf{z} \| \boldsymbol{\mu}_{p}
$$

At an electron rotating in the "equator" plane of proton, we can introduce the following notation for field components and the rotation parameters:

$$
\begin{aligned}
\boldsymbol{\beta} & \equiv \beta_{\vartheta}=\frac{v_{\vartheta}}{c} \\
\boldsymbol{H} & \equiv H_{z} \\
\boldsymbol{E} & \equiv E_{r}
\end{aligned}
$$

Between positively charged proton and negatively charged electron must exist a force of Coulomb attraction ([5], \$24):

$$
\boldsymbol{F}_{e}=\operatorname{e\gamma }\{\boldsymbol{E}-[\boldsymbol{\beta} \times \boldsymbol{H}]\}
$$

In addition to the Coulomb force $F_{e}$, a Lorentz force will act on the rotating electron. This power is due to the impact of the proton magnetic moment $\mu_{p}$. An observer moving in a magnetic field $\mathrm{H}$ "sees" in its system the electric field ([5], §24, Equation (24.2)):

$$
\boldsymbol{E}_{L}=\left[\boldsymbol{\beta} \times \frac{\boldsymbol{H}+[\boldsymbol{\beta} \times \boldsymbol{E}]}{\sqrt{1-\beta^{2}}}\right],
$$

which gives rise to the Lorentz force:

$$
\boldsymbol{F}_{L}=e \boldsymbol{E}_{L}
$$

Where the electric field intensity

$$
\boldsymbol{E}=E_{r}=\frac{e}{R_{0}^{2}} \frac{\boldsymbol{R}_{0}}{R_{0}} .
$$

The magnetic field is created by magnetic moment of proton

$$
\boldsymbol{H}_{p}=\gamma \frac{\boldsymbol{\mu}_{p}}{R_{0}^{3}} .
$$

In equilibrium, the attraction forces $F_{e}$ and $F_{L}$ will be balanced by the centrifugal force:

$$
\boldsymbol{F}_{c}=\frac{\gamma m_{e} c^{2} \beta^{2}}{R_{0}} \frac{\boldsymbol{R}_{0}}{R_{0}}
$$

and by a force tending to break the current ring:

$$
\boldsymbol{F}_{b}=\frac{e \beta \mu_{0}}{R_{0}^{3}} .
$$

where $\mu_{p}$ and $\mu_{0}$ are magnetic moments of proton and current ring.

Thus, we obtain the equation that determines the balance 


$$
e \gamma\left(\frac{e}{R_{0}^{2}}+\beta \gamma \frac{\mu_{p}}{R_{0}^{3}}\right)+e \gamma \beta\left(\gamma \frac{\mu_{p}}{R_{0}^{3}}-\beta \frac{e}{R_{0}^{2}}\right)=\gamma \beta^{2} \frac{m_{e} c^{2}}{R_{0}}+\frac{e \beta \mu_{0}}{R_{0}^{3}} .
$$

In a result this equilibrium equation with unknown $R_{0}$ and $\beta$ becomes:

$$
\frac{R_{0}}{\alpha r_{c}}=\left(\frac{1}{\beta^{2}}-1+2 \frac{\mu_{p}}{\beta e R_{0}}-\frac{\mu_{0}}{\beta \gamma e R_{0}}\right) \text {. }
$$

where $\alpha=\frac{e^{2}}{\hbar c}$ is the fine structure constant,

$$
\begin{aligned}
& r_{c}=\frac{\hbar}{m_{e} c} \text { is the Compton radius, } \\
& \mu_{0}=\frac{e R_{0} \beta}{2} \text { is magnetic moment of the circular electron current. }
\end{aligned}
$$

To record the second equation relating these parameters, we can use the virial theorem. According to this theorem, the kinetic energy of system of particles, combined by electromagnetic interaction at their finite motion, is equal to half of their potential energy, taken with the opposite sign:

$$
\mathcal{E}_{\text {kin }}=-\frac{e}{2}(\varphi+\beta A)=\frac{e}{2}\left\{\frac{e}{R_{0}}+\frac{\beta}{\sqrt{1-\beta^{2}}} \frac{\mu_{p}}{R_{0}^{2}}-\frac{\mu_{0}}{R_{0}^{2}}\right\}
$$

On the other hand, according to the definition, the kinetic energy of a relativistic electron:

$$
\mathcal{E}_{\text {kin }}=m_{e} c^{2}\left(\frac{1}{\sqrt{1-\beta^{2}}}-1\right) .
$$

So the second equation linking these parameters, takes the form:

$$
m_{e} c^{2}\left(\frac{1}{\sqrt{1-\beta^{2}}}-1\right)=\frac{1}{2}\left\{\frac{e^{2}}{R_{0}}-\frac{e \mu_{0}}{R_{0}^{2}}+\frac{\beta}{\sqrt{1-\beta^{2}}} \frac{e \mu_{p}}{R_{0}^{2}}\right\} .
$$

Solving Equation (23) and Equation (26) together, we have:

$$
R_{0} \approx 0.3808 \alpha r_{c} \approx 1.0732 \times 10^{-13} \mathrm{~cm}
$$

and

$$
\beta \approx 0.92448
$$

\subsection{The Main Parameters of Neutron}

These options allow to determine the magnetic moment of the neutron, its mass and energy of decay.

Generalized angular momentum (spin) of the current ring (spin) (see [3], Section 2.3)

$$
\boldsymbol{s}_{0}=\left[\boldsymbol{p}_{0} \times \boldsymbol{R}_{0}\right]=0 .
$$

The magnetic moment of the current ring, expressed in nuclear magneton $\mu_{N}$

$$
\xi_{0}=\frac{e R_{0} \beta}{2 \mu_{N}} \approx-4.7176 \text {. }
$$


Therefore, the sum of proton magnetic moment and the magnetic moment of ring

$$
\xi_{p}+\xi_{0}=2.793-4.7176=-1.9246 \text {. }
$$

This value is consistent with the measured value of the magnetic moment of neutron $\left(\xi_{n}=-1.91304272\right)$ :

$$
\frac{\xi_{p}+\xi_{0}}{\xi_{n}} \approx 1.0061
$$

The sum of masses of proton and relativistic electron is consistent with measured mass of neutron:

$$
\frac{m_{p}+\frac{m_{e}}{\sqrt{1-\beta^{2}}}}{m_{n}}=1.0001 .
$$

According to the virial theorem, the total energy of a particles system is equal to its kinetic energy Equation (24):

$$
\mathcal{E}_{\text {kin }}=1.329 \times 10^{-6} \text { erg }=829 \mathrm{keV} .
$$

At the decay of neutron, this energy must go into the kinetic energy of the emitted electron (and antineutrino). It is consistent with the experimentally determined boundary of the spectrum of decay electrons $\mathcal{E}=782 \mathrm{keV}$.

\subsection{Discussion}

In the above model of proton composed of quarks with integral charges, there is no question of observability of quarks in a free state.

However, there is a series of unknown. Generally, it is not clear why quark-positron and quark-electron does not annihilate and what interactions cause them to unite in a completely stable particle - proton, which decays in nature is not observed.

This consent of estimates and measured data indicates that the neutron is not an elementary particle. It should be seen as a some relativistic analog of the Bohr hydrogen atom. With the difference: a non-relativistic electron in the Bohr atom forms a shell by means of Coulomb forces and in neutron the relativistic electron is held generally by the magnetic interaction too ([3] [4]).

According to the Gilbert's principle, a theoretical construct can be attributed to reliably established if it is confirmed by experimental data. Therefore the measurement confirmation for the discussed above electromagnetic model of neutron is the most important, required and completely sufficient argument of its credibility. Nevertheless, it is important for the understanding of the model to use the standard theoretical apparatus at its construction. It should be noted that for the scientists who are accustomed to the language of relativistic quantum physics, the methodology used for the above estimates does not contribute to the perception of the results at a superficial glance. It is commonly thought that for the reliability, a consideration of an affection of relativism on the electron behavior in the Coulomb field should be carried out within the Dirac theory. However that is not necessary in the case of calculating of the neutron 
mass, magnetic moment and its decay energy. In this case, all relativistic effects described by the terms with coefficients $\left(1-\frac{v^{2}}{c^{2}}\right)^{-1 / 2}$ compensate each other and completely fall out. The neutron considered in our model is the quantum object. Its radius $R_{0}$ is proportional to the Compton radius and hence depends on the Planck constant $\hbar$. But it can not be considered as relativistic particle, because coefficient $\left(1-\frac{v^{2}}{c^{2}}\right)^{-1 / 2}$ is not included in the definition of $R_{0}$. In the particular case of the calculation of the mass, magnetic moment of the neutron and the energy of its decay, it allows to find an equilibrium of the system from the balance of forces, as it can be made in the case of non-relativistic objects. The another case exits at the evaluation of the neutron lifetime. The relativism affects on this parameter apparently and one can not obtain even a correct estimation of the order of its value.

\section{The One-Electron Bond between Two Protons}

\subsection{The Molecular Hydrogen Ion}

The quantum-mechanical model of simplest molecule-the molecular hydrogen ionwas first formulated and solved by Walter Heitler and Fritz London in 1927 [6]. They calculated the attraction that arises between two protons through the electron exchange, when their state can be described by two-well potential (Figure 3). This exchange is the quantum-mechanical effect and its classical counterpart does not exist. (Some of the details of this calculation are presented in [3] [4]).

The main conclusion of this work is that the binding energy between the two protons, which arises due to electron exchange, on the order of magnitude is close to the binding energy of proton and electron (energy of the electron in the first Bohr orbit).

This conclusion is in relatively good agreement with the measurement data. The measurement result is different from the calculation in less than two times.

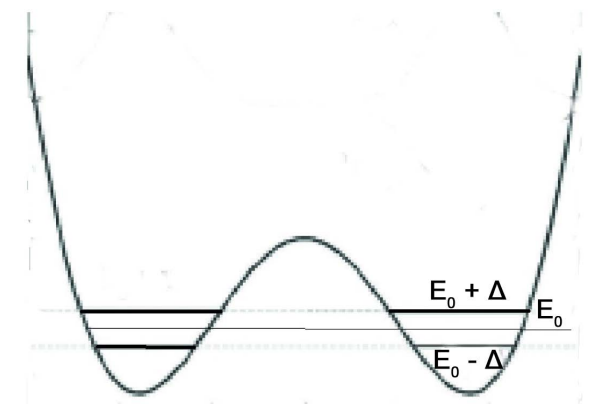

Figure 3. The schematic representation of the potential well with two symmetric states. In the ground state, electron can be either in the right or in the left hole. In the unperturbed state, its wave functions are either $\varphi_{1}$ or $\varphi_{2}$ with the energy $E_{0}$. The quantum tunneling transition from one state to another leads to the splitting of energy level and to the lowering of the sublevel on $\Delta$. 


\subsection{Deutron and Other Light Nuclei}

\subsubsection{Deutron}

The electromagnetic model of the neutron, discussed above, provides a new look at the mechanism of interaction of neutron and proton. Neutron-i.e. proton, surrounded by a relativistic electron cloud-plus free proton together constitute an object similar to the molecular hydrogen ion. The difference is that now electron is relativistic, the radius of its orbit $R_{0} \approx 10^{-13} \mathrm{~cm}$ and its mass of about $2.25 \mathrm{~m}_{e}$.

The application of the results of quantum-mechanical Heitler-London calculations for this case makes it possible to evaluate the binding energy of the deuteron with an accuracy of approximately the same as in the case of the hydrogen molecular ion ([3] [4]). The estimation gives the binding energy approximately $\Delta \approx 2.13 \times 10^{-6} \mathrm{erg}$, whereas measurement of this energy gives

$$
\mathcal{E}_{d} \approx 3.567 \times 10^{-6} \text { erg. }
$$

\subsubsection{Light Nuclei}

Nucleus ${ }_{2}^{3} \mathrm{He}$

As can be seen from the schematic structure of this nucleus (Figure 4), its binding energy is composed by three pairwise interacting protons. Therefore it can be assumed that it equals to the tripled energy of deuteron:

$$
\mathcal{E}_{\text {Нез }}=3 \times \mathcal{E}_{d} \approx 10.70 \times 10^{-6} \mathrm{erg} .
$$

The mass defect of this nucleus

$$
\Delta M(\text { He3 })=3 M_{p}+m_{e *}-M_{\text {Hе3 }}=1.19369 \times 10^{-26} \mathrm{~g} .
$$

Thus mass defect corresponds to the binding energy

$$
\mathcal{E}_{\Delta M(\mathrm{He} 3)}=\Delta M(\mathrm{He} 3) \times \mathrm{C}^{2} \approx 10.73 \times 10^{-6} \mathrm{erg} .
$$

Consent energies $\mathcal{E}_{\text {Нез }}$ and $\mathcal{E}_{\triangle M(\text { Нез })}$ can be considered as very good.

Nucleus ${ }_{2}^{4} \mathrm{He}$

As can be seen from the schematic structure of this nucleus (Figure 4), its binding energy is composed by six pairwise interacting protons which are realised by two electrons. On this reason its binding energy can be considered as:

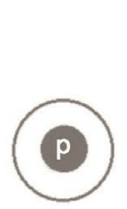

$\mathbf{n}$

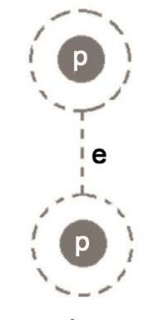

d

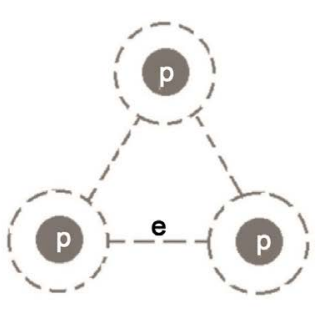

He-3

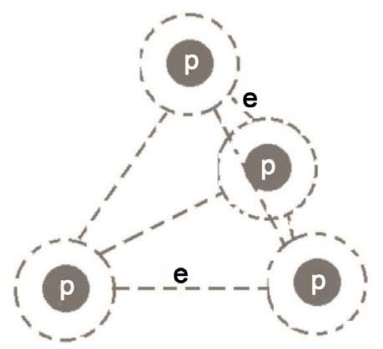

$\mathrm{He}-4$

Figure 4. The schematic representation of the structure of light nuclei. Dotted lines schematically indicate the possibility of a relativistic electron hopping between protons. 


$$
\mathcal{E}_{\text {He } 4}=2 \times 6 \times \mathcal{E}_{d} \approx 42.80 \times 10^{-6} \mathrm{erg} .
$$

The mass defect of this nucleus

$$
\Delta M(\mathrm{He} 4)=4 M_{p}+2 m_{e^{*}}-M_{\mathrm{He} 4}=48.62 \times 10^{-26} \mathrm{~g} .
$$

Thus mass defect corresponds to the binding energy

$$
\mathcal{E}_{\Delta M(\mathrm{He} 4)}=\Delta M(\mathrm{He} 4) \times c^{2} \approx 43.70 \times 10^{-6} \text { erg. }
$$

Consent of these energies can be considered as alright.

Nucleus ${ }_{3}^{6} \mathrm{Li}$

The binding energy of Li- 6 can be represented by the sum of binding energy of He-4 and deuteron. The last placed on next shell and must have a weak bounding with He-4:

$$
\mathcal{E}_{\text {Li6 }} \approx \mathcal{E}_{\mathrm{He} 4}+\mathcal{E}_{d} \approx 47.26 \times 10^{-6} \mathrm{erg} .
$$

The mass defect of this nucleus

$$
\Delta M(L i 6)=6 M_{p}+3 m_{e^{*}}-M_{L i 6}=54.30 \times 10^{-26} \mathrm{~g},
$$

and corresponding binding energy

$$
\mathcal{E}_{\Delta M(L i 6)}=\Delta M(\operatorname{Li6}) \times C^{2} \approx 48.80 \times 10^{-6} \mathrm{erg},
$$

That really confirms the weak link between the protons in different shells.

It should be noted that the situation with the other light nuclei are not so simple.

The nucleus ${ }_{1}^{3} \mathrm{~T}$ consists of three protons and two communicating electrons between them. Jumps of two electrons in this system should obey to the Pauli exclusion principle. Apparently this is the reason that the binding energy of tritium is not very much greater than the binding energy of He-3.

Nuclear binding energy of Li-7 can be represented as $\mathcal{E}_{\text {Li7 }} \approx \mathcal{E}_{\text {He4 }}+\mathcal{E}_{T}$. But it is quite a rough estimate. At that the binding energy of unstable nucleus Be- 8 very precisely equal to twice binding energy of $\mathrm{He}-4$.

\subsection{Discussion}

The good agreement between the calculated binding energy of some light nuclei with measured data suggests that nuclear forces (at least in the case of these nuclei) have the above-described exchange character. These forces arise as a result of a purely quantum effect of exchange relativistic electrons.

For the first time the attention on the possibility of explaining the nuclear forces based on the effect of electron exchange apparently drew I.E. Tamm [7] back in the 30s of the last century. However, later the model of the $\pi$-meson and gluon exchange becomes the dominant in nuclear physics. The reason for that is clear. To explain the magnitude and range of the nuclear forces need particle with a small wavelength. Nonrelativistic electrons does not fit it. However, on the other hand, models $\pi$-meson or gluon exchange was not productive: It gives not possibility to calculate the binding energy of even light nuclei.

Therefore, the simple assessment of the binding energy given above and consistent 
with measurements is the clear proof that the so-called strong interaction (in the case of light nuclei) is a manifestation of the quantum-mechanical effect of attraction between protons produced by the relativistic electron exchange.

\section{Neutrino}

Previously, it was shown that in the standard Maxwell's electromagnetic field theory, there are two possibilities [4]. Different excitation methods can initiate in free space (empty ether) or a transverse electromagnetic wave (photon), or magnetic $\gamma$-quantum (magnetic soliton)-a clot of wave devoid of the electric component.

An electromagnetic wave may be caused by oscillating electric or magnetic dipoles.

According to Maxwell's equations, the electric field of photon is proportional to the second time derivative of the time-varying magnetic moment which generates photon:

$$
\boldsymbol{E}(R, t)=-\frac{1}{c^{2} R}\left[\ddot{\boldsymbol{m}}\left(\boldsymbol{t}^{*}\right) \times \boldsymbol{n}\right] .
$$

The magnetic field has two components. The first component is exactly equal to the electric field value but turned in space on $\pi / 2$. The second component depends from first derivative of the time-varying magnetic moment and much less them the first one (in $\lambda / R$ times):

$$
\boldsymbol{H}(R, t)=-\frac{1}{c^{2} R}\left[\boldsymbol{n} \times\left[\ddot{\boldsymbol{m}}\left(t^{*}\right) \times \boldsymbol{n}\right]\right]+\frac{1}{c R^{2}}\left[\boldsymbol{n} \times\left[\dot{\boldsymbol{m}}\left(t^{*}\right) \times \boldsymbol{n}\right]\right]
$$

There is another ether excitation method: a magnetic moment of elementary particle is created or disappear almost instantaneously as a result of the nuclear reaction. This magnetic blow over ether excites magnetic oscillations in it. If the time dependence of the magnetic moment is described by a sharp Heaviside's step-function, the first derivative of this step-function is $\delta$-function and the second derivative is zero. Therefore, when the leading edge of the step-function lasts about $10^{-23}$ second (this is the assessment of the transformation time of $\pi$-meson into $\mu$-meson at which antineutrinos arises) should be emitted photon having $\delta$-shaped magnetic component and devoid of the electric component (see in detail in [4]).

Characteristic features of the magnetic soliton is that, being circularly polarized, it must have spin $\hbar / 2$, and its interaction with matter nearly two dozen orders of magnitude weaker than that of the electromagnetic wave. This feature is due to the fact that in nature there are no magnetic monopoles.

This suggests that the magnetic $\gamma$-quantum can be identified with neutrino. At the same time the birth of a magnetic moment occurs antineutrinos and neutrinos in its disappearance.

So in the process of successive transformation of $\pi$-meson at first in $\mu$-meson, and then in electron, such magnetic $\gamma$-quanta arise three times (Figure 5).

\section{Mesons}

In the chain of transformations pion $\rightarrow$ muon $\rightarrow e$, three neutrinos are born (Figure 5). Charged pions have spins equal to zero and do not have magnetic dipoles. At the time 


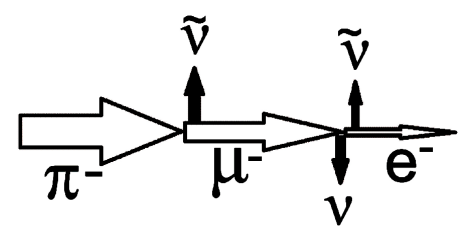

Figure 5. Scheme of production of three magnetic $\gamma$-quanta (neutrinos) in the process of decay $\pi^{-}$-meson. $\pi^{-}$-meson does not have a magnetic moment. It turns at the decay into $\mu^{-}$-meson carrying a magnetic moment. This process should be accompanied by a magnetic blow on ether and emission of magnetic $\gamma$-quantum (departure antineutrino). In the decay of $\mu^{-}$-meson, its magnetic moment disappears and another magnetic $\gamma$-quantum (neutrino) emitted. The third magnetic soliton (antineutrino) emits at the moment of an electron arising.

of conversion of $\pi^{-}$-meson in $\mu^{-}$-meson, magnetic moment arises abruptly (during approximately $10^{-23} \mathrm{~s}$ ), which is accompanied by the emission of antineutrino $\tilde{v}$. At the decay of the muon, neutrino generates, which is caused by the fact that the muon magnetic moment disappears. Electron with its magnetic moment is born at the same time. This leads to the emission of the antineutrino $\tilde{v}$. The fact that other products, except neutrinos and antineutrinos, do not arise in these reactions, leads us to the assumption that the pion and muon are not independent elementary particles, but they are excited states of electron.

These mesons have masses

$$
\begin{aligned}
M_{\pi}^{ \pm} & =273.13 m_{e} \\
M_{\mu}^{ \pm} & =206.77 m_{e}
\end{aligned}
$$

Let us assume that the excited state of the electron is formed due to the fact that a point particle with mass $M=\frac{m_{e}}{\sqrt{1-\beta^{2}}}$ and with charge -e rotates in a circle of radius R with velocity $v \rightarrow c$. We will assume that stable excited states will be those for which the de Broglie wavelength is placed on the circumference of an integer times:

$$
\frac{2 \pi R}{\lambda_{D}}=n
$$

where $\lambda_{D}=\frac{2 \pi \hbar}{p}$ is the de Broglie wavelength, $n=1,2,3, \cdots$ is integer.

At that the invariant kinetic angular momentum (spin) of particle

$$
\boldsymbol{s}=n\left[\left(\boldsymbol{p}-\frac{e}{c} \boldsymbol{A}\right) \times \boldsymbol{R}\right]
$$

where $\boldsymbol{p}=\gamma m_{e} c \boldsymbol{\beta}$ is mechanical momentum of particle,

$\boldsymbol{A}=\frac{[\boldsymbol{\mu} \times \boldsymbol{R}]}{R^{3} \sqrt{1-\beta^{2}}}$ is the vector potential of the magnetic field generated by the rotating charge.

Considering the magnetic moment of gyrating charge $e$

$$
\boldsymbol{\mu}=\frac{e}{2 c}[\boldsymbol{v} \times \boldsymbol{R}]
$$


we obtain

$$
s=n \hbar\left(1-\frac{\alpha}{2 \sqrt{1-\beta^{2}}}\right),
$$

where $\alpha=\frac{e^{2}}{\hbar c}$ is the fine structure constant.

\subsection{Excited State with $s=0$}

According to Equation (51) the condition $s=0$ corresponds to such particle velocity at which the coefficient $\frac{1}{\sqrt{1-\beta^{2}}}$ is equal to $2 / \alpha$. The mass of the particle, because of its relativistic increasing, is equal to

$$
M_{0}=\frac{2}{\alpha} m_{e}=274.08 m_{e} .
$$

This value is very close to the mass of $\pi^{ \pm}$-meson Equation (47), which spin is zero:

$$
\frac{M_{0}}{M_{\pi^{ \pm}}} \simeq 1.003
$$

\subsection{Excited State with $n=2$ and $s=\hbar / 2$.}

At $n=2$ and $s=\hbar / 2$, the coefficient $\frac{1}{\sqrt{1-\beta^{2}}}$ must be equal to $3 / 2 \alpha$. At that mass of the particle, because of its relativistic increasing, is equal to

$$
M_{1 / 2}=\frac{3}{2 \alpha} m_{e}=205.56 m_{e} .
$$

This value is very close to the mass of $\mu^{ \pm}$-meson (spin $\hbar / 2$ ):

$$
\frac{M_{1 / 2}}{M_{\mu^{ \pm}}} \simeq 0.9941
$$

The possibility to calculate meson masses, basing only on their spins, confirms the assumption that these mesons are not elementary particles but simple excited states of electron.

\section{Conclusions}

The ability to calculate basic properties of different elementary particles detects the failure of the quark model with fractional charges of quarks in which such assessments can not be obtained.

The Gell-Mann's quark model in its present form demonstrates the possibility of classifying the particles, but it does not prove that such classification is the only one possible and correct.

It is important to note that for the description of proton-neutron interactions (in light nuclei) there is no need to involve a model of gluons, and use theories of strong 
and weak interactions.

Indeed, the exchange of relativistic electron in deuteron as well as the exchange of non-relativistic electron in a hydrogen molecular ion is pure quantum mechanical phenomenon. There is no reason to consider it as a special strong interaction of Nature.

The emission of neutrinos occurs in the $\beta$-decay (or K-capture). The process of disintegration of the nuclei as $\alpha$ - and $\beta$-decays does not require to introduce any special fundamental interactions. But $\beta$-decay has a significant feature: at it magnetic moment of free electron occurs at extremely short time (or disappear when the K-capture). This produces a magnetic blow on ether and leads to the emission of magnetic $\gamma$-quantum, ie neutrino. The nature of this phenomenon is purely electromagnetic and there is no needs to enter a special weak or electro-weak interaction for its describing.

However, there is no proving do not introduce other interactions at descriptions of more complex microcosm objects. It is obvious that at calculation of nuclear forces in heavy nuclei, one needs to take into account other effects associated with the existence of nuclear shells.

Nevertheless, the possibility of the electromagnetic description of some of particles makes it relevant the question of correctness of existing descriptions of many other more complex objects of microcosm.

Obviously, the validation of such description must be based on experimental test data of basic properties of these objects. A successful method of systematization of particles in a certain table can not be considered conclusive evidence of the correctness and uniqueness of this approach. As Gilbert argued, the theoretical construction must be obligatorily confirmed by the measurement data.

\section{References}

[1] Gilbert, W. (1600) De magneto magneticisque corporibus et de magno magnete tellure. London.

[2] Vasiliev, B.V. (2015) Research \& Reviews. Journal of Pure and Applied Physics, 3, 2. http://www.rroij.com/open-access/on-the-disservice-of-theoretical-physics-work-on-the-b ugs.php?aid=61047

[3] Vasiliev, B.V. (2015) About Nature of Nuclear Forces Journal of Modern Physics, 6, 648659. http://www.scirp.org/Journal/PaperInformation.aspx?PaperID=55921

[4] Vasiliev, B.V. (2015) International Journal of Modern Physics and Application, 3, 25-38. http://www.aascit.org/journal/archive2?journalId=909\&paperId=3935

[5] Landau, L.D. and Lifshitz, E.M. (1971) The Classical Theory of Fields (Volume 2 of A Course of Theoretical Physics). Pergamon Press, NY.

[6] Heitler, W. and London, F. (1927) Zeitschrift fur Physik, 44, 455-472. http://dx.doi.org/10.1007/BF01397394

[7] Tamm, I.E. (1934) Nature, 134, 1011. http://dx.doi.org/10.1038/1341010c0 
Submit or recommend next manuscript to SCIRP and we will provide best service for you:

Accepting pre-submission inquiries through Email, Facebook, LinkedIn, Twitter, etc. A wide selection of journals (inclusive of 9 subjects, more than 200 journals)

Providing 24-hour high-quality service

User-friendly online submission system

Fair and swift peer-review system

Efficient typesetting and proofreading procedure

Display of the result of downloads and visits, as well as the number of cited articles

Maximum dissemination of your research work

Submit your manuscript at: http://papersubmission.scirp.org/

Or contact jmp@scirp.org 